\title{
Osteopathic principles for basic scientists
}

IRVIN M. KORR, Ph.D

Fort Worth, Texas

In more than 40 years of close association with the osteopathic medical profession, as teacher and researcher, I have had ample opportunity to observe the demonstration of osteopathic principles at work as guides to clinical practice. In that long period, I have also had the opportunity, as a recipient of osteopathic care, to draw the implications of those principles, to my health and that of my family, and to find in them, a richly rewarding way of life. Further, I have found that my personal and continually evolving interpretations of, and elaborations on, those principles, from a physiologist's viewpoint, have also been valuable guides in both teaching and research.

The purpose of this brief essay is to share these interpretations and elaborations with my colleagues in the biomedical sciences in the colleges of osteopathic medicine, for whatever value they may find in them. It would serve its purpose even if it only stimulated my colleagues to formulate their own interpretations, implications, and elaborations. It would be even better if it also helped in the design of teaching programs, in the choice of priorities to be taught within each discipline, and in the formulation of new fundamental questions for research.

\section{The unity of the person}

The original principle, "the body is a unit," emphasized that every part of the body is in communication with all other parts, and that a change in any part elicits adaptive changes in all other parts and, therefore, in the body as a whole. I think that this principle needs to be extended beyond the body to the person and to body/mind unity and interplay. For the modern bioscientist, body/mind interaction in health and disease needs no defense. The mechanisms underlying the influences of mind on body and of body on mind, of which the diverse neuropep- tides and neuroendocrine mechanisms are but two examples, are being investigated in laboratories throughout the world.

The person is the environment in which parts exist and operate. Every quality and circumstance of the person's life influences the quality of function of every cell, tissue, organ, and system. Given the unique features of human life, as distinguished from other animal life, it is inevitable that the circumstances under which the parts operate, and the demands made upon them in uniquely human life, are vastly different from those under which the body components evolved. The implications of these "new" circumstances and demands have hardly begun to be explored. The influence of these differences on the body mechanisms, and on the ways in which they contribute to the unique features of human life (and human frailty), are well within the domain of the basic medical sciences.

Just as the human context differs from other organismic contexts, so does every human differ from all other humans. In short, there is an infinite variety of ways of being human (and "normal"), and an infinite variety of idiosyncratic influences on fundamental biological functions and processes. Not only initial genetic endowment, but also every other aspect of that individual life, including the mental, emotional, behavioral, physical, social, and environmental, enters into conditioning the quality of every component and process. The study of human diversity-from the molecular to the organismic-is, therefore, also within the realm of fundamental biomedical science.

This, however, requires an important recognition: that although the concept of the heart, the stomach, or the adrenals may be useful for teaching purposes, these are but abstractions and generalizations about idealized norms. In reality, there are only hearts, stomachs, and adrenals, each dif- 
ferent from all others according to whom it serves, and according to the way in which it has been required to serve in the life of that individual. It is these differences in personal context that will eventually explain why some hearts (or other organs or processes) become impaired in different ways at different ages, while others go on functioning for a century or more. The fundamental mechanisms underlying the reciprocal influence of life course on bodily functions, and of bodily functions on life course, are in need of exploration.

It is recognized that inclusion of the human context, in physiological, biochemical, immunological, pharmacological, anatomical, or other biomedical research, may be misconstrued as rejection of the prevailing assumption in biomedical research: That the best way (perhaps the only way) to understand man and the nature of his life is to take him apart and study the component parts and processes, minutely and exhaustively; that when we have identified each part and shown how it operates (even when it has been extracted from other animals), then we shall understand man. Inclusion of the human context should, however, be viewed not as denial of the (unquestionable) value of the reductionist paradigm, but as recognition of its incompleteness. Placing it in the human context accomplishes the needed completion.

A simple analogy may help to clarify this point. In the course of achieving our current knowledge and understanding of water, it has been undeniably important to know that it is composed of hydrogen and oxygen in a certain relationship. However, even the most minute study of hydrogen and oxygen, and of their bonds, would not have yielded the properties of water-the three states, the boiling and freezing points, the surface tension, and viscosity, or the fact that the specific gravity of the solid state is less than that of the liquid state. That is, if one wishes to understand water, one must study water. Similarly, as Alexander Pope said, "the proper study of mankind is man." The proper study of human physiology, biochemistry, pathology, health, and disease, is also man.

Unquestionably, new paradigms will need to be developed which are no less rigorous, no less "scientific" than the reductionist approach and its experimental designs. This would be an enormous contribution to the completion of another of the "scientific revolutions," of which Thomas Kuhn has written so eloquently.

\section{Vis medicatrix naturae}

This ancient Latin phrase, originally designating the body's self-healing capacity, today represents the better understood complex of homeostatic, regulatory, protective, regenerative, and compen- satory mechanisms with which every human is endowed. Their unimpaired operation is collectively manifest in the maintenance and restoration of health and the healing of wounds.

Clearly, scientific investigation of these mechanisms and components is inseparable from the factors that affect them, both favorably and unfavorably. Included among the factors are those that are peculiar to humans and human life and that vary from person to person. The appearance of new disciplines, such as psychoneuroimmunology and study of the relation of the neuropeptides and other of the "body's own medicines" (endocoids) to human mood, attitude, expectations, and behavior, reflect the increasing recognition of the relevance of the human factors to fundamental questions in physiology, biochemistry, immunology, pharmacology, and the other basic sciences.

There are many other factors that influence the efficacy of the body's healing mechanisms. Perhaps the most important contribution of osteopathic medicine is that its focus is on the control of the most strategic (and responsive) of the factors, on behalf of the patient's health.

\section{The primacy of the musculoskeletal system}

The traditional emphasis of medicine on the viscera tends to convey the impression that human activity-behavior of the person-is a composite of visceral functions, such as myocardial contraction, peristalsis, digestion, vasomotion, and glomerular filtration. The fact is that the ultimate instrument of human action and behavior is the musculoskeletal system. Every human act consists of coordinated contractions and relaxations of skeletal muscles, most of them activating bony levers. Human behavior is, ultimately, the continually changing patterns of muscular contractions and relaxations.

The musculoskeletal system is the means through which we express our humanity and individuality, our intellect, every feeling, belief, hope, and fear, and every ethical, moral-and even scientific-principle. None has overt expression and value except insofar as it is acted out-by the musculoskeletal system.

The "acting-out" is, of course, organized by the central nervous system, in accordance with sensory input and cerebral control. Indeed, most of the efferent output of the CNS is directed to this sytem, and most of the sensory input to the CNS is from the musculoskeletal system. In short, the nervous system is very much preoccupied with motion.

Similarly, the functions and processes of other systems of the body-circulatory, respiratory, digestive, metabolic-are also largely involved in meeting the logistical requirements of the mus- 
culoskeletal system. As the most massive of all the systems and the greatest energy consumer, the body's musculature places enormous, continuous, and rapidly changing demands upon other systems for fuels, replacements of degraded cellular components, removal of metabolic products, dissipation of heat, and control of the celluar environment.

In short, the total economy of the body is continually "tuned" to the high and variable requirements of the musculoskeletal system. The sympathetic division of the autonomic nervous system seems "responsible" for the moment-to-moment adjustment of visceral, circulatory, and metabolic processes, according to changing muscular activity (and environmental circumstances). The parasympathetic division, on the other hand, seems responsible for long-term adjustments according to customary activity (and usual environment) of the individual. It is evident, therefore, that in its varying activities, the musculoskeletal system is a continual source of challenge and perturbation of the dynamic balance of the homeostatic systems and of the constancy of the internal milieu.

While profoundly influencing and placing demands upon the other systems, the musculoskeletal system, in turn, is profoundly influenced by all others and by the adequacy of their responses to its requirements. Illness results from-indeed, is-disparity between those requirements and the degree to which they are met by the other systems. Given a significant disparity, motion becomes difficult or impossible, and therapuetic rest is automatically enforced. Such disparities (and therefore illness) may be due to inappropriate or excessive demand, inadequate vis- ceral, circulatory or metabolic response, aberrant communication between the musculoskeletal and other systems, or combinations thereof.

Because of its unique and relatively recently evolved vertical stance (while still bearing, as Darwin said, "the indelible stamp of its lowly origin"), the human framework is vulnerable to the compressive forces of gravity. "Somatic dysfunction" frequently develops in the human musculoskeletal system, in the course of individual and lifelong adaptations to the relentless force of gravity. Somatic dysfunctions may, depending on the other circumstances in the individual life, become impediments to the competence of other systems, for they, too, are communicated to other systems. The strategic importance of somatic dysfunction to health is that it exaggerates the impact of other negative factors in the person's life. Because of the two-way communication, the musculoskeletal system not only influences, but also reflects the quality of function or pathology of other organs and systems. Thus, it provides a basis for diagnosis, treatment, and prevention-in which osteopathic manipulative medicine plays a crucial role.

It is hoped that this interpretation of osteopathic philosophy may be helpful to biological scientists, in the fulfillment of their opportunities and obligations as members of osteopathic medical faculties.

Dr. Korr is a professor of medical education at the Texas College of Osteopathic Medicine, Fort Worth, Texas.

Dr. Korr, Camp Bowie at Montgomery, Fort Worth, Texas 76107. 


\section{OSTEOPATHIC RESEARCH: GROWTH AND DEVELOPMENT \\ - Where have we been? \\ - Where are we now? \\ - Where are we going?

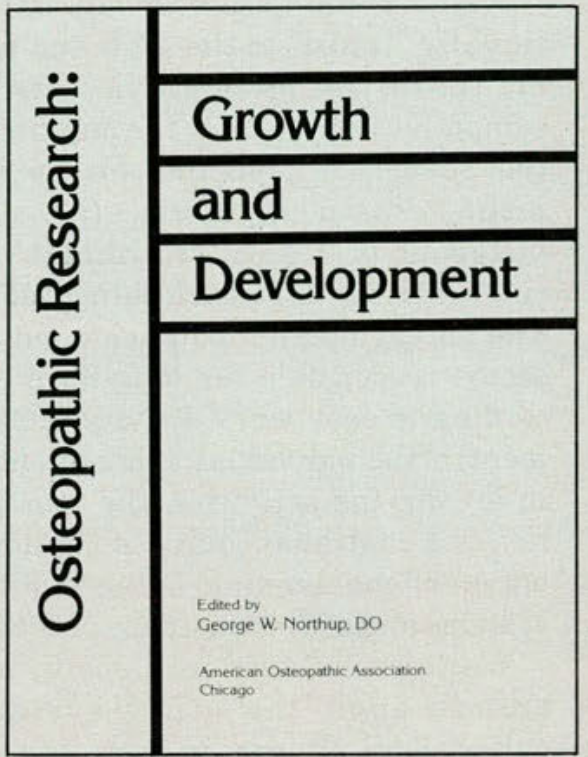

- General History -Wilbur V. Cole, DO

- Anatomical and Histopathological Evidence

-Wilbur V. Cole, DO

- Biomechanics-Myron C. Beal, DO

- Physiology —Albert F. Kelso, PhD

- Clinical Research-Myron C. Beal, DO

- Status and Future of Osteopathic Research

-Albert F. Kelso, PhD

Alexandra A. Townsend, DO

An excellent source for students, teachers, researchers, and practitioners.

Send check and order form to:

American Osteopathic Association

142 E. Ontario Street

Chicago, Illinois 60611

Attn: Order Department

Please send me: hardcover copies of Osteopathic Research: Growth and Development (\$11 each) 